\title{
ERRATUM
}

G.C. Donaldson $\cdot$ M. Scarborough $\cdot$ K. Mridha

L. Whelan - M. Caunce $\cdot$ W.R. Keatinge

\section{Effect of posture on body temperature of young men in cold air}

Eur J Appl Physiol (1996) 73: 326-331

In lines 10-11 of the Abstract (p. 326) an unfortunate error occurred: "temperature differences should read "temperatures". 